\title{
Transport Through a Driven Three-Site System
}

\author{
Michael Strass \\ Sigmund Kohler \\ Jörg Lehmann \\ Peter Hänggi
}

Institut für Physik, Universität Augsburg Universitätsstraße, Augsburg, Germany

We study the current and its noise properties of the electron transport through a molecular wire modeled by a three tight-binding levels. Within a high-frequency approximation, the time-dependent transport problem is described by a static problem with renormalized parameters. The analytical results are in good agreement with an exact numerical solution.

Keywords: driven systems; noise; quantum transport

PACS: 05.60.Gg; 85.65.+h; 05.40. $-\mathrm{a} ; 72.40 .+\mathrm{w}$

\section{INTRODUCTION}

In recent years several suggestions how to set up a single electron transistor have been made $[1,2,3,4]$. The development of new methods to contact molecules or molecule-like systems and control the electron transport through them $[5,6,7]$ is a major issue in the promising field of molecular electronics [8,9]. The conventional setup of such a transistor requires a gate in order to trigger via the gate voltage the current from the source to the drain which correspond to the lead contacts of the molecular system. In fact, the implementation of a single gate electrode which creates a sufficiently strong field at the molecule is a

We thank Sébastien Camalet and Gert-Ludwig Ingold for helpful discussions. This work has been supported by the Volkswagen-Stiftung under Grant No. I/77 217 and the DFG Sonderforschungsbereich 486.

Address correspondence to Peter Hänggi, Institut für Physik, Universität Augsburg, Universitätsstraße 1, D-86135 Augsburg, Germany. Tel.: 0049-831-598-3250, Fax: 49821-598-3222, E-mail: hanggi@physik.uni-augsburg.de 
demanding task $[2,10,11]$. Instead of using the static fields of a gate electrode, oscillating external fields or an oscillating gate voltage might be a convenient alternative to control the electrical current according to a molecular field-effect transistor. Depending on the nanosystem itself, laser fields or microwave radiation are appropriate for this purpose. As has been shown recently in a theoretical work [12], a switch can be obtained by applying a coherent monochromatic field. Moreover, it is even possible to control the corresponding noise level [13].

In the present paper we study current and noise through a tightbinding system consisting of three sites which are subject to an external ac driving. This model describes both molecular wires under the influence of laser fields and coherently coupled quantum dot systems [14] irradiated by microwaves. In Section 3, analytical expressions for the current and zero-frequency noise are derived for the static case. Turning on an external field, we demonstrate in Section 4 that the driven system can be approximated in the high-frequency regime by the static system with renormalized parameters. The article is rounded off by comparing the analytic expressions with the numerical results of Ref. [13].

\section{THE MODEL}

We consider the setup depicted in Figure 1 which consists of three sites subject to an external oscillating field and coupled to two leads. It is described by the time-dependent Hamiltonian

$$
H(t)=H_{\text {system }}(t)+H_{\text {leads }}+H_{\text {contacts }} .
$$

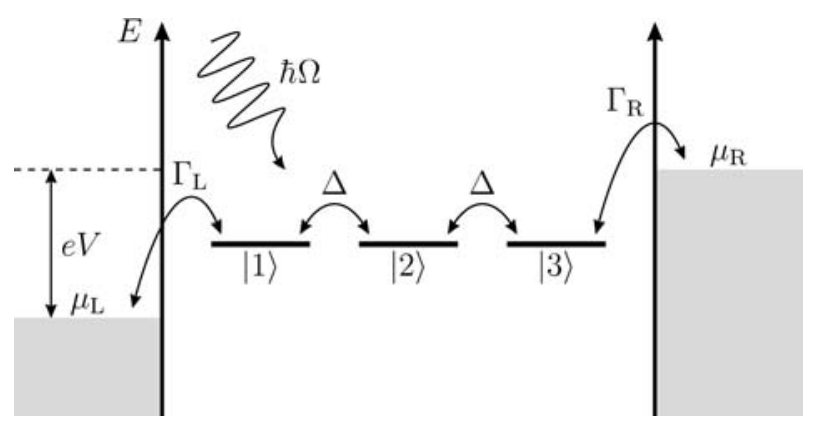

FIGURE 1 Nanoscale conductor consisting of three sites. The terminating sites are coupled with $\Gamma_{\mathrm{L}}$ and $\Gamma_{\mathrm{R}}$ to the respective lead with chemical potentials $\mu_{\mathrm{L}}$ and $\mu_{\mathrm{R}}=\mu_{\mathrm{L}}+e V$, whereas the intrasite coupling is described by a hopping matrix element $\Delta$. 
The Hamiltonian

$$
H_{\text {system }}(t)=-\Delta\left(c_{1}^{\dagger} c_{2}+c_{2}^{\dagger} c_{1}+c_{2}^{\dagger} c_{3}+c_{3}^{\dagger} c_{2}\right)+A\left(c_{1}^{\dagger} c_{1}-c_{3}^{\dagger} c_{3}\right) \cos (\Omega t)
$$

represents the driven three-site system in the tight-binding description disregarding electron-electron and electron-phonon interactions. The fermion operators $c_{n}$ and $c_{n}^{\dagger}$ annihilate and create, respectively, an electron at the level $n$. Expressed by the second term of the Hamiltonian (2), the system itself is exposed to an ac field with frequency $\Omega=2 \pi / \tau$. This field results in a dipole force shifting the on-site energies periodically. The amplitude $A$ is proportional to the component of the electric field strength parallel to the system axis. The dipole approximation is well justified since the wavelength of the external field we consider in this work is some orders of magnitudes larger than the system size of the nanoconductor. Moreover, the coupling of the nearest neighbor sites is given by a hopping matrix element $\Delta$. The electron reservoirs of the leads are described by the Hamiltonian

$$
H_{\text {leads }}=\sum_{\ell, q} \varepsilon_{\ell q} c_{\ell q}^{\dagger} c_{\ell q}
$$

where $c_{\mathrm{L} q}^{\dagger}\left(c_{\mathrm{R} q}^{\dagger}\right)$ creates a electron in the left (right) lead with momentum $q$. For simplicity spin is neglected. The electron distribution in the leads is assumed to be grand-canonical with inverse temperature $\beta=1 / k_{B} T$ and electro-chemical potential $\mu_{\mathrm{L} / \mathrm{R}}$. An applied voltage $V$ corresponds to $\mu_{\mathrm{R}}-\mu_{\mathrm{L}}=e V$, where $-e$ is the electron charge. Finally, the contact between the terminating sites and the leads results in the tunneling Hamiltonian

$$
H_{\text {contacts }}=\sum_{q}\left(V_{\mathrm{L} q} c_{\mathrm{L} q}^{\dagger} c_{1}+V_{\mathrm{R} q} c_{\mathrm{R} q}^{\dagger} c_{3}\right)+\text { h.c. }
$$

Since we are not interested in the effects that stem from the microscopic structure of the tunneling processes, we consider in the following the system-lead coupling to be energy-independent. In this so-called wide band limit the spectral density is assumed constant, i.e., $\Gamma_{\ell}(E)=2 \pi \sum_{q}\left|V_{\ell q}\right|^{2} \delta\left(E-\varepsilon_{\ell q}\right)=\Gamma_{\ell}$.

\section{TRANSPORT WITHOUT AC-DRIVING}

As a starting point, we derive expressions for current and noise for the static system, setting $A=0$ in the Hamiltonian (2). Solving the Heisenberg equations of motion for the lead operators, we obtain 


$$
c_{\mathrm{L} q}(t)=c_{\mathrm{L} q}\left(t_{0}\right) \mathrm{e}^{-\mathrm{i} \varepsilon_{\mathrm{Lq}}\left(t-t_{0}\right) / \hbar}-\frac{\mathrm{i} V_{\mathrm{L} q}}{\hbar} \int_{t_{0}}^{t} \mathrm{~d} t^{\prime} \mathrm{e}^{-\mathrm{i} \varepsilon_{\mathrm{Lq}}\left(t-t^{\prime}\right) / \hbar} c_{1}\left(t^{\prime}\right)
$$

and a corresponding expression for $c_{\mathrm{R} q}(t)$ with $\mathrm{L}$ replaced by $\mathrm{R}$ and $c_{1}$ by $c_{3}$. Inserting (5) into the Heisenberg equations of motion of the three-site system and exploiting the wide-band limit, one arrives at

$$
\begin{aligned}
\dot{c}_{1 / 3} & =\frac{\mathrm{i}}{\hbar} \Delta c_{2}-\frac{\Gamma_{\mathrm{L} / \mathrm{R}}}{2 \hbar} c_{1 / 3}+\xi_{\mathrm{L} / \mathrm{R}}(t), \\
\dot{c}_{2} & =\frac{\mathrm{i}}{\hbar} \Delta c_{1}+\frac{\mathrm{i}}{\hbar} \Delta c_{3} .
\end{aligned}
$$

The operator-valued Gaussian noise defined as

$$
\xi_{\ell}(t)=-\frac{\mathrm{i}}{\hbar} \sum_{q} V_{\ell q}^{*} \exp \left(-\frac{\mathrm{i}}{\hbar} \varepsilon_{\ell q}\left(t-t_{0}\right)\right) c_{\ell q}\left(t_{0}\right)
$$

has within the grand-canonical ensemble the properties

$$
\begin{aligned}
\left\langle\xi_{\ell}(t)\right\rangle & =0, \\
\left\langle\xi_{\ell}^{\dagger}(t) \xi_{\ell^{\prime}}\left(t^{\prime}\right)\right\rangle & =\delta_{\ell \ell^{\prime}} \frac{\Gamma_{\ell}}{2 \pi \hbar^{2}} \int \mathrm{d} \varepsilon \mathrm{e}^{\mathrm{i} \varepsilon\left(t-t^{\prime}\right) / \hbar} f_{\ell}(\varepsilon) .
\end{aligned}
$$

Here, $f_{\ell}(\varepsilon)=\left\{1+\exp \left[\beta\left(\varepsilon-\mu_{\ell}\right)\right]\right\}^{-1}$ denotes the Fermi function with chemical potential $\mu_{\ell}, \ell=\mathrm{L}, \mathrm{R}$.

The time-dependent current through the contact $\ell$ is defined as $I_{\ell}(t)=e(\mathrm{~d} / \mathrm{d} t) \sum_{q} c_{\ell q}^{\dagger} c_{\ell q}$. Making use of Eq. (5), the current through the left contact becomes in the wide-band limit

$$
I_{\mathrm{L}}(t)=\frac{e}{\hbar} \Gamma_{\mathrm{L}} c_{1}^{\dagger}(t) c_{1}(t)-e\left\{c_{1}^{\dagger}(t) \xi_{\mathrm{L}}(t)+\xi_{\mathrm{L}}^{\dagger}(t) c_{1}(t)\right\} .
$$

The operator $c_{1}(t)$ is obtained by solving Eqs. (6) in the stationary limit $t_{0} \rightarrow-\infty$ using the Green function method. After the elimination of the backscattering terms, we finally obtain

$$
I=\left\langle I_{\mathrm{L}}\right\rangle=\frac{e}{2 \pi \hbar} \int \mathrm{d} E\left[f_{\mathrm{R}}(E)-f_{\mathrm{L}}(E)\right] T(E),
$$

with the transmission $T(E)=\Gamma_{\mathrm{L}} \Gamma_{\mathrm{R}}\left|G_{13}(E)\right|^{2}$, where $G_{13}(E)$ denotes the corresponding matrix element of the Green function. For a three-site system with symmetric coupling to the leads, i.e., $\Gamma_{\mathrm{L}}=\Gamma_{\mathrm{R}}=\Gamma$, the transmission reads

$$
T(E)=\frac{\Gamma^{2} \Delta^{2}}{\left|(2 \mathrm{i} E+\Gamma)\left(2 \mathrm{i} E^{2}+E \Gamma-4 \mathrm{i} \Delta^{2}\right)\right|^{2}} .
$$


The noise of the current across contact $\ell$ is given by the symmetric auto-correlation function of the current fluctuation operator $\Delta I_{\ell}(t)=I_{\ell}(t)-\left\langle I_{\ell}(t)\right\rangle$. The noise strength measured in experiments is characterized by its zero-frequency component $S=S(\omega=0)=(1 / 2)$ $\int_{-\infty}^{+\infty} \mathrm{d} t\left\langle\Delta I_{\ell}(t) \Delta I_{\ell}(0)+\Delta I_{\ell}(0) \Delta I_{\ell}(t)\right\rangle$, which is independent of the contact $\ell$ due to total charge conservation. It is convenient to express $S$ in terms of the transmission function $T(E)$ as [15]

$$
\begin{aligned}
S=\frac{e^{2}}{2 \pi \hbar} \int \mathrm{d} E & \left\{(E)\left[f_{\mathrm{L}}(E)\left[1-f_{\mathrm{L}}(E)\right]+f_{\mathrm{R}}(E)\left[1-f_{\mathrm{R}}(E)\right]\right]\right. \\
+ & \left.T(E)[1-T(E)]\left[f_{\mathrm{R}}(E)-f_{\mathrm{L}}(E)\right]^{2}\right\} .
\end{aligned}
$$

For zero temperature, the first line vanishes while the second line describes pure shot noise, which has its physical origin in the discreteness of the charge carriers.

In the following, we consider voltages much larger than all other energy scales of the setup indicated by the subscript $\infty$ for the discussed quantities. As a consequence, the current noise will entirely be due to shot noise. Furthermore, in this limit, the results for current and noise will not depend on temperature. In the expression (10) for the current, the difference of the Fermi distributions then practically equals one for energies where the transmission is non-vanishing. The current thus reads

$$
I_{\infty}=\frac{e}{2 \pi \hbar} T=\frac{e \Gamma}{2 \hbar} \frac{\Delta^{2}}{\Delta^{2}+(\Gamma / 2)^{2}},
$$

where $T=\int \mathrm{d} E T(E)$ is the total transmission. With the same argument we find from (12) for the current noise

$$
S_{\infty}=\frac{e^{2} \Gamma}{\hbar} \frac{\Delta^{2}\left(\Gamma^{4}-4 \Gamma^{2} \Delta^{2}+16 \Delta^{4}\right)}{\left(4 \Delta^{2}+\Gamma^{2}\right)^{3}} .
$$

A very illustrative quantity is the relative noise strength characterized by the so-called Fano factor $F=S / e I$ [16] and which becomes in our case

$$
F_{\infty}=\frac{\Gamma^{4}-4 \Gamma^{2} \Delta^{2}+16 \Delta^{4}}{2\left(4 \Delta^{2}+\Gamma^{2}\right)^{2}} .
$$

Analyzing the Fano factor (15) as a function of the ratio of the tunneling matrix element $\Delta$ and the level width $\Gamma$, we find the minimal Fano 
factor $F_{\infty}=1 / 8$ for $\Delta=\Gamma / 2$. In terms of transport properties, this means that the channel is optimally transparent. For weak systemlead coupling $\Gamma \ll \Delta$, the two contacts between the terminating sites and the leads form the limiting step of the transport process. We effectively arrive at transport through a double-barrier system with a Fano factor $F_{\infty}=1 / 2$ [17]. The same Fano factor is obtained in the opposite limit $\Gamma \gg \Delta$, but for a different physical reason: now the double-barrier is established at the central cite since the left and right ones hybridize with the adjacent lead.

\section{HIGH-FREQUENCY REGIME}

Now we switch on the external driving. It has been shown $[18,19]$ that for large driving frequencies $\Omega$, the time-dependent system can be approximated by a static one with renormalized parameters. The starting point of this approximation scheme is the unitary transformation

$$
U_{0}(t)=\exp \left(-\mathrm{i} \frac{A}{\hbar \Omega}\left(c_{1}^{\dagger} c_{1}-c_{3}^{\dagger} c_{3}\right) \sin (\Omega t)\right),
$$

which we first apply to the system Hamiltonian (2). For sufficiently large driving frequencies $\Omega \gg \Delta / \hbar$, we can separate time scales. Thereby, we neglect fast oscillations of the transformed Hamiltonian by averaging over a driving period [20,21]. Finally, we arrive at the effective system Hamiltonian

$$
\begin{aligned}
\bar{H}_{\text {system }} & =\frac{1}{\tau} \int_{0}^{\tau} \mathrm{d} t\left(U_{0}^{\dagger} H_{\text {system }}(t) U_{0}-\mathrm{i} \hbar U_{0}^{\dagger} \dot{U}_{0}\right) \\
& =-\Delta_{\text {eff }}\left(c_{1}^{\dagger} c_{2}+c_{2}^{\dagger} c_{1}+c_{2}^{\dagger} c_{3}+c_{3}^{\dagger} c_{2}\right),
\end{aligned}
$$

which is of the same form as in the static case but with $\Delta$ replaced by the effective hopping matrix element $\Delta_{\text {eff }}=J_{0}(A / \hbar \Omega) \Delta[12,21-24] . J_{0}$ is the zeroth order Bessel function of the first kind. Thus, $\Delta_{\text {eff }}$ is controllable via the driving parameters and can even vanish. The transformation (16) affects the system-lead coupling, as well. If we apply $U_{0}(t)$ also to $H_{\text {contacts }}$ and solve the Heisenberg equations of motion for the lead and system operators in the wide-band limit as we did in Section 3, we can eventually extract the new fluctuation operators. For the left lead one finds

$$
\eta_{\mathrm{L}}(t)=-\frac{\mathrm{i}}{\hbar} \sum_{q} V_{\mathrm{L} q}^{*} \exp \left[-\frac{\mathrm{i}}{\hbar}\left(\varepsilon_{\mathrm{L} q}\left(t-t_{0}\right)-\frac{A}{\Omega} \sin (\Omega t)\right)\right] c_{\mathrm{L} q}\left(t_{0}\right),
$$


which obeys the correlation function

$$
\begin{aligned}
\left\langle\eta_{\mathrm{L}}^{\dagger}(t) \eta_{\mathrm{L}}\left(t^{\prime}\right)\right\rangle= & \frac{\Gamma_{\mathrm{L}}}{2 \pi \hbar^{2}} \int \mathrm{d} \varepsilon \sum_{k, k^{\prime}} \mathrm{e}^{\mathrm{i} \varepsilon\left(t-t^{\prime}\right) / \hbar} f_{\mathrm{L}}(\varepsilon+k \hbar \Omega) \\
& \times J_{k}(A / \hbar \Omega) J_{k^{\prime}}(A / \hbar \Omega) \mathrm{e}^{-\mathrm{i}\left(k-k^{\prime}\right) \Omega t}
\end{aligned}
$$

and corresponding expressions for the right lead. Again we neglect the fast oscillating contributions by time-averaging. Then, the correlation function is the same as in the static case, cf. Eq. (8), but with the Fermi function replaced by the effective distribution function

$$
f_{\ell, \mathrm{eff}}(E)=\sum_{k=-\infty}^{\infty} J_{k}^{2}(A / \hbar \Omega) f_{\ell}(E+k \hbar \Omega)
$$

Physically, the $k$ th summand in this term describes the absorption $(k<0)$ or emission $(k<0)$ of $|k|$ photons by an electron of energy $E$ which is transferred from lead $\ell$ to the system. These processes are weighted by the square of the $k$ th order Bessel function of the first kind. An example for the effective distribution functions for a small transport voltage is plotted in Figure 2.

Two situations depending on the voltage have to be distinguished. For sufficiently large voltages and in the relevant energy regime of non-vanishing transmission, the left and right effective distribution functions are zero and one, respectively. The transport properties are determined by the transmission (11), the current (13) and zerofrequency noise (14) derived in the previous section for the static case but with the replacement $\Delta \rightarrow \Delta_{\text {eff }}$. We denote the current and the noise

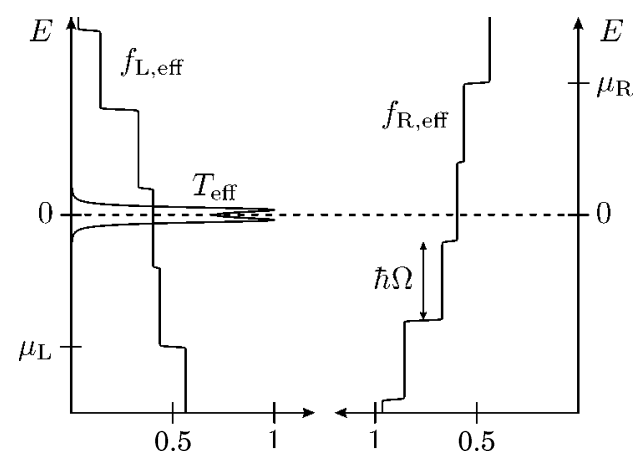

FIGURE 2 Left and right effective distribution function and effective transmission function for a typical set of parameters at room temperature. 
in this limit by $\bar{I}_{\infty}$ and $\bar{S}_{\infty}$, respectively. The effective hopping matrix element is zero for certain driving parameters and consequently in the high-frequency limit, transport vanishes.

The case of a finite voltage has to be considered with more care. Since the effective transmission $T_{\text {eff }}(E)$ illustrated in Figure 2 is peaked around $E=0$ in the high-frequency regime, we can replace $f_{\ell, \mathrm{eff}}(E)$ in the current and noise expressions by its value at $E=0$ which leaves us with $f_{\ell, \text { eff }}(0)=\sum_{k<\mu_{\ell} / \hbar \Omega} J_{k}^{2}(A / \hbar \Omega)$. The time-averaged current and the zero-frequency noise are thus given by

$$
\begin{gathered}
\bar{I}=\bar{I}_{\infty} \sum_{|k| \leq K(V)} J_{k}^{2}(A / \hbar \Omega), \\
\bar{S}=\frac{e}{2} \overline{\boldsymbol{I}}_{\infty}+\left(\sum_{|k| \leq K(V)} J_{k}^{2}(A / \hbar \Omega)\right)^{2}\left(\overline{\boldsymbol{S}}_{\infty}-\frac{e}{2} \overline{\boldsymbol{I}}_{\infty}\right),
\end{gathered}
$$

where $K(V)$ denotes the largest integer not exceeding $e V / 2 \hbar \Omega$. Note that these expressions are just valid if the voltage $V$ is not close to a multiple of $\hbar \Omega$ and consequently the transmission peak does not appear close to a step at $\mu_{\ell}+k \hbar \Omega$ of the effective distribution function. In that case, $f_{\ell, \text { eff }}(0)$ would not be constant in the vicinity of $E=0$ any more. Also worth mentioning is the fact that in contrast to the static situation, the result (22) contains contributions stemming from the first line in the noise expression (12) even in the zero-temperature limit.

\section{RESULTS AND CONCLUSION}

The comparison of the numerically exact result and the highfrequency approximation for the time-averaged current, the zerofrequency noise and the Fano factor is shown in Figure 3. The exact results (solid line) are computed within the Floquet approach of Ref. [13] as a function of the driving amplitude $A$ for $\mu_{R}=-\mu_{L}=24 \Delta$ which corresponds to a relatively large voltage. Besides the results for this finite voltage in the high-frequency regime (dashed line), also the curves for infinite voltage are depicted (dotted line) in order to get a notion of the influence of the applied voltage.

The agreement of the current and the noise between exact results and high-frequency approximation is very good for the chosen set of parameters. Although the approximated noise starts to deviate from the Floquet approach for amplitudes $A>60 \Delta$, we can explain the characteristic current and noise suppressions of the numerically exact result with help of the high-frequency approximation. These suppressions appear whenever the effective hopping matrix element $\Delta_{\text {eff }}$ 


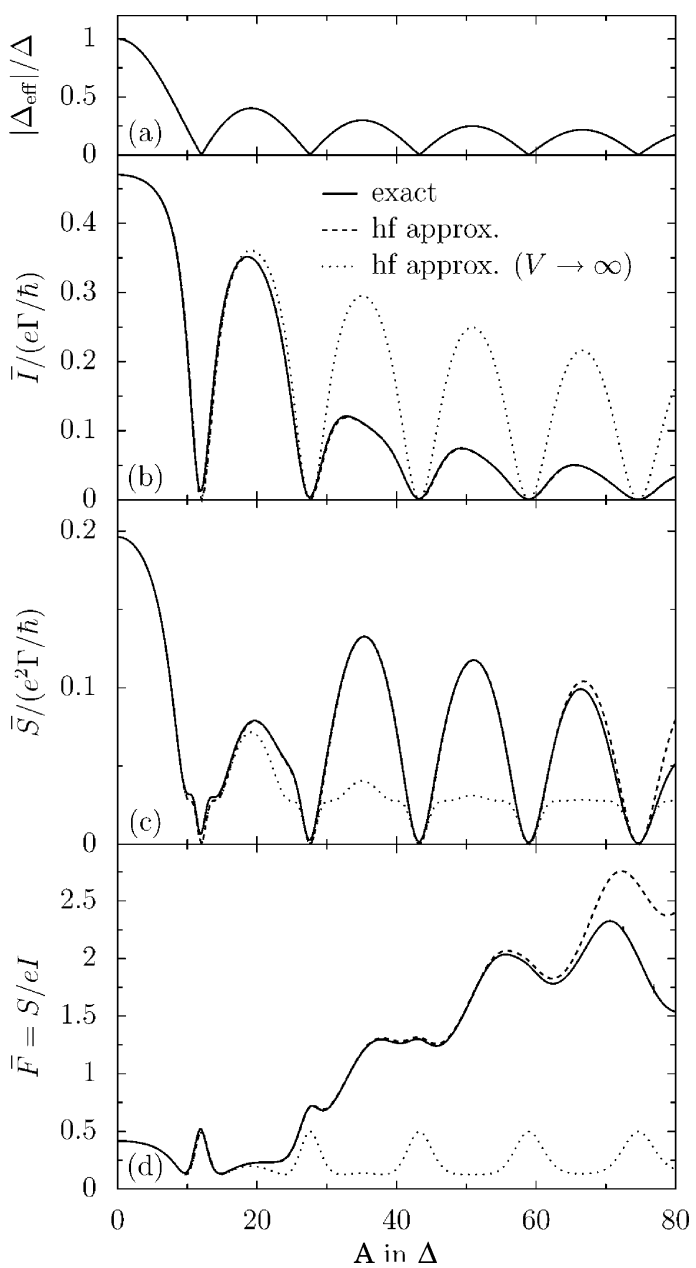

FIGURE 3 (a) Effective hopping matrix element $\left|\Delta_{\text {eff }}\right|$, (b) time-averaged current $\bar{I}$, (c) zero-frequency noise $\bar{S}$, and (d) Fano factor $F=\bar{S} / e \bar{I}$ as a function of the driving amplitude $A$ in units of $\Delta$. The used parameters are the voltage $V=48 \Delta / e$, the coupling strength $\Gamma=0.5 \Delta$ and the driving frequency $\Omega=5 \Delta / \hbar$. Depicted are the numerically exact results (solid lines), the highfrequency (hf) results (21) and (22) for finite voltage (dashed lines), and the infinite voltage results (13) and (14) with the replacement $\Delta \rightarrow \Delta_{\text {eff }}$ (dotted lines).

vanishes. In this case, the ratio $A / \hbar \Omega$ corresponds to a zero of the Bessel function $J_{0}$ depicted in Figure 3(a). What actually happens in the system by varying $A$ is that the system-lead coupling is tuned 
depending on the ratio of $\Gamma$ to $\Delta_{\text {eff }}$ as discussed in the static situation after Eq. (15). Therefore, starting from very small amplitudes, the Fano factor has the value $F \approx 1 / 2$ describing tunneling through a double-barrier. For larger amplitudes when $\Gamma \approx \Delta_{\text {eff }}, F$ reaches a minimum and optimal transport is established. Note however that for the exact result, the current and noise are not totally zero at the suppressions in contrast to the high-frequency approximation. If we raise $A$ further, we obtain again $F \approx 1 / 2$ since the situation is essentially that of a single site weakly coupled to two leads [15].

For $A<e V / 2$, the current and the noise are well approximated by their asymptotic values for infinite voltage, $\bar{I} \approx \overline{\boldsymbol{I}}_{\infty}$ and $\bar{S} \approx \bar{S}_{\infty}$, since $J_{k}(x) \approx 0$ for $x>k$ and $\sum J_{k}^{2}(x)=1$. The Fano factor behaves different from the static case $i^{k}$ the regime of larger amplitudes and even exceeds one half in the finite voltage limit.

The control of electron transport through a system with discrete levels by means of a time-periodic field is a tempting concept for future devices in nano-electronics. Interestingly enough, an external driving allows one to manipulate both the current and its noise level characterized by the Fano factor. We gained profound physical insight by applying a high-frequency approximation: The driving essentially renormalizes the parameters of the static setup. In particular, the tunnel matrix elements between adjacent sites can be suppressed by the external field and, thus, it is possible to switch between strong and weak effective system-lead coupling. This explains in turn quantitatively the observed noise reduction.

\section{REFERENCES}

[1] Aviram, A. \& Ratner, M. A. (1974). Chem. Phys. Lett., 29, 277.

[2] Liang, W., Shores, M. P., Bockrath, M., Long, J. R., \& Park, H. (2002). Nature, 417, 725.

[3] Heath, J. R. \& Ratner, M. A. (2003). Physics Today, 56, 43.

[4] Metzger, R. M. (2003). Chem. Rev., 103, 3803.

[5] Reed, M. A., Zhou, C, Muller, C. J., Burgin, T. P., \& Tour, J. M. (1997). Science, 278, 252.

[6] Cui, X. D., Primak, A., Zarate, X., Tomfohr, J., Sankey, O. F., Moore, A. L., Moore, T. A., Gust, D., Harris, G., \& Lindsay, S. M. (2001). Science, 294, 571.

[7] Reichert, J., Ochs, R., Beckmann, D., Weber, H. B., Mayor, M., \& von Löhneysen, H. (2002). Phys. Rev. Lett., 88, 176804.

[8] Hänggi, P., Ratner, M., \& Yaliraki, S. (2002). Chem. Phys., 281, 111-502. Special Issue: Processes in Molecular Wires.

[9] Nitzan, A. \& Ratner, M. A. (2003). Science, 300, 1384.

[10] Zhitenev, N. B., Meng, H., \& Bao, Z. (2002). Phys. Rev. Lett., 88, 226801.

[11] Lee, J. -O., Lientschnig, G., Wiertz, F., Struijk, M., Janssen, R. A. J., Egberink, R., Reinhoudt, D. N., Hadley, P., \& Dekker, C. (2003). Nano Lett., 3, 113. 
[12] Lehmann, J., Camalet, S., Kohler, S., \& Hänggi, P. (2003). Chem. Phys. Lett., 368, 282.

[13] Camalet, S., Lehmann, J., Kohler, S., \& Hänggi, P. (2003). Phys. Rev. Lett., 90, 210602.

[14] Blick, R. H., Haug, R. J., Weis, J., Pfannkuche, D., von Klitzing, K., \& Eberl, K. (1996). Phys. Rev. B, 53, 7899.

[15] Blanter Ya. M. \& Büttiker, M. (2000). Phys. Rep., 336, 1.

[16] Fano, U. (1947). Phys. Rev., 72, 26.

[17] Chen L. Y. \& Ting, C. S. (1991). Phys. Rev. B, 43, 4534.

[18] Kohler, S., Camalet, S., Strass, M., Lehmann, J., Ingold, G.-L., \& Hänggi, P. (2004). Chem. Phys., 296, 243.

[19] Camalet, S., Kohler, S., \& Hänggi, P. (2004). cond-mat/0402182.

[20] Großmann, F., Jung, P., Dittrich, T., \& Hänggi, P. (1991). Z. Phys. B, 84, 315.

[21] Grifoni, M. \& Hänggi, P. (1998). Phys. Rep., 304, 229.

[22] Grossmann, F., Dittrich, T., Jung, P., \& Hänggi, P. (1991). Phys. Rev. Lett., 67, 516.

[23] Großmann, F. \& Hänggi, P. (1992). Europhys. Lett., 18, 571.

[24] Platero, G. \& Aguado, R. (2004). Phys. Rep., 395, 1. 